\title{
Positionally Selective Growth of Embryonic Spinal Cord Neurites on Muscle Membranes
}

\author{
H. Wang, ${ }^{1}$ S. R. Chadaram, ${ }^{1}$ A. S. Norton, ${ }^{1}$ R. Lewis, ${ }^{2}$ J. Boyum, ${ }^{1}$ W. Trumble, ${ }^{1}$ J. R. Sanes, ${ }^{2}$ and \\ M. B. Laskowski ${ }^{1}$ \\ 1WWAMI Medical Program, University of Idaho, Moscow, Idaho 83844-4207, and 2Department of Anatomy and \\ Neurobiology, Washington University School of Medicine, St. Louis, Missouri 63110
}

\begin{abstract}
Motor neurons from distinct positions along the rostrocaudal axis generally innervate muscles or muscle fibers from corresponding axial levels. These topographic maps of connectivity are partially restored after denervation or transplantation under conditions in which factors of timing and proximity are eliminated. It is therefore likely that motor neurons and some intramuscular structures bear cues that bias synapse formation in favor of positionally matched partners. To localize these cues, we studied outgrowth of neurites from embryonic spinal cord explants on carpets of membranes isolated from perinatal rat muscles. Neurites from rostral (cervical) and caudal (lumbar) spinal cord slices exhibit distinct growth preferences. In many instances, rostrally derived neurites grew selectively on membranes from forelimb muscles or from a single thoracic muscle
\end{abstract}

(the serratus anterior) when given a choice between these membranes and membranes from hindlimb muscles or laminin. Caudally derived neurites almost never exhibited such rostral preferences, but instead preferred membranes from hindlimb muscles or a single hindlimb muscle (the gluteus) to rostral muscles or laminin. Likewise, spinal neurites exhibited distinct position-related preferences for outgrowth on membranes of clonal myogenic cell lines derived from specific rostral and caudal muscles. Taken together these results suggest that the membranes of motor axons and myotubes bear complementary labels that vary with rostrocaudal position and regulate neuromuscular connectivity.

Key words: motor neurons; neurites; laminin; ephrins; Eph kinases; spinal cord; selective growth
Motor neurons form topographic maps of connectivity with skeletal muscles and muscle fibers. Individual muscles arise from segmentally arranged somites and are generally innervated by the axons of motor neurons whose cell bodies reside in corresponding spinal levels. Topography is also present within many individual muscles: the rostrocaudal axis of each motor pool maps systematically onto the muscle's rostrocaudal axis. (Swett et al., 1970; Brown and Booth, 1983a,b; Bennett and Lavidis, 1984a,b; Laskowski and Sanes, 1987; Laskowski and Owens, 1994). An obvious possibility is that these orderly patterns arise as consequences of proximity and timing. For example, axons growing through rostral ventral roots will contact rostral rather than caudal muscles as they arrive in the periphery. Even within muscles, coordinated programs of axon outgrowth and myogenesis could account for the formation of maps.

Perhaps surprisingly, several experimental results provide compelling evidence that in addition to proximity and timing, molecular recognition plays a role in forming maps of neuromuscular connectivity. First, when muscles are transplanted from distinct axial levels to a common site, they are selectively reinnervated by axons corresponding to their position of origin (Wigston and Sanes, 1985). Second, topographic maps are present within mus-

\footnotetext{
Received Dec. 10, 1998; revised March 15, 1999; accepted April 5, 1999.

This work was supported by grants from National Institutes of Health (M.B.L., J.R.S.) and the Muscular Dystrophy Association (J.R.S.).

We thank Dr. Lea Ziskind-Conhaim for help in initial experiments with spinal cord slices. We also thank Drs. Joseph Cloud and Mark DeSantis for providing tissue culture facilities. We appreciate the technical assistance of Irma Laskowski and Suzanna Hueble, and the gift of CNTF from Regeneron Pharmaceuticals.

Correspondence should be addressed to Dr. Michael B. Laskowski, WWAMI Medical Program, University of Idaho, Moscow, ID 83844-4207.

Copyright (C) 1999 Society for Neuroscience $\quad 0270-6474 / 99 / 194984-10 \$ 05.00 / 0$
}

cles that are supplied by a single peripheral nerve within which axons from multiple spinal segments are intermingled randomly (Laskowski and Sanes, 1987). Third, in some muscles, maps are formed or sharpened by a completely intramuscular process of synapse elimination after connections have formed (Brown and Booth, 1983a,b; Bennett and Lavidis, 1984a,b). Fourth, intramuscular maps are partially restored during reinnervation after nerve damage, although all regenerating axons have equal and simultaneous access to the muscle (Brown and Hardman, 1987; Hardman and Brown, 1987; Laskowski and Sanes, 1988; DeSantis et al., 1992; Grow et al., 1995; Laskowski et al., 1998a). Together, these results suggest that motor neurons and some intramuscular structures bear complementary cues that vary with rostrocaudal position and bias synapse formation in favor of positionally matched partners. To date, however, the molecular identity of these cues and even the cellular location of the intramuscular cues remain unknown.

The best-studied topographic map of neural connectivity is that formed by retinal axons on the optic tectum (superior colliculus) (Sperry, 1963). Recently, exciting progress has been made in identifying some of the cellular interactions and molecular cues that underlie mapping. Much of this progress is based on elegant studies by Bonhoeffer and colleagues (Walter, et al., 1987a,b), using a bioassay in which explants from defined portions of the retina were laid on a substrate of tectal membrane fragments. The membranes were taken from defined tectal loci and arranged in alternating stripes. Axons showed position-dependent outgrowth preferences in this "stripe" assay (Walter et al., 1987a), analysis of which led to the realization that inhibitory cues can pattern projections (Walter et al., 1987b; Cox et al., 1990; Stahl et al., 1990). Eventually this led to the identification of ephrins and Eph 
kinases as mediators of some inhibitory interactions that underlie mapping along the anterior-posterior axis (Friedman and O'Leary, 1996; Drescher et al., 1995; Flanagan and Vanderhaeghen, 1998; Frisen et al., 1998).

In light of the striking parallels between topographic mapping in the retinotectal and neuromuscular systems (Sanes, 1993), we adapted Bonhoeffer's stripe assay for use in investigating the localization and nature of intramuscular cues that guide axons. Using this assay, we show here that embryonic spinal cord neurites grow selectively on membranes of muscles derived from corresponding rostrocaudal positions. We also show that neurites make position-dependent choices between membranes of immortalized myogenic cells derived from individual muscles. Results from the cell lines show not only that myotube membranes bear position-dependent cues that axons recognize, but they show that these cell lines also provide a suitable source for identification of the molecules that mediate these interactions.

\section{MATERIALS AND METHODS}

Membrane isolation. Timed-pregnant female Sprague Dawley rats (Simonsen, Gilroy, CA) were deeply anesthetized with ketamine-xylazine or chloral hydrate when the embryos had reached day 18 of gestation (E18), and a laparotomy was performed. Embryos were removed, decapitated, and placed in ice-cold sterile dissecting solution containing (in $\mathrm{mm}): 139 \mathrm{NaCl}, 5 \mathrm{KCl}, 1.1 \mathrm{MgCl}_{2}, 4 \mathrm{CaCl}_{2}, 4.2 \mathrm{HEPES}, 11$ glucose, and $0.3 \mathrm{mg} / \mathrm{ml}$ penicillin, $0.1 \mathrm{mg} / \mathrm{ml}$ streptomycin, $\mathrm{pH} 7.2$. Muscles from the forelimbs and hindlimbs of each embryo were removed, frozen in liquid nitrogen and stored at $-80^{\circ} \mathrm{C}$. Neonatal (P0) muscles were similarly prepared and stored.

To prepare a membrane-rich fraction, muscles were homogenized with a Polytron at $70 \%$ maximal speed for $3 \times 30 \mathrm{sec}$, in $2-5 \mathrm{vol}$ of "homogenizing buffer" (10 mM Tris- $\mathrm{HCl}, 1.5 \mathrm{mM} \mathrm{CaCl}_{2}, 50 \mu \mathrm{M}$ leupeptin, $1 \mu \mathrm{M}$ aprotinin, $2 \mu \mathrm{M}$ pepstatin $\mathrm{A}, \mathrm{pH}$ 7.4). The homogenate was filtered through four layers of cheesecloth, placed on a $5-50 \%$ sucrose gradient, and centrifuged at $50,000 \times g$ for $10 \mathrm{~min}$. Crude membranes were collected from the interface, washed once with modified PBS $(136 \mathrm{~mm}$ $\mathrm{NaCl}, 2.6 \mathrm{~mm} \mathrm{KCl}, 8.1 \mathrm{~mm} \mathrm{Na} \mathrm{HPO}_{4}, 1.4 \mathrm{~mm} \mathrm{NaH}_{2} \mathrm{PO}_{4}, 0.68 \mathrm{~mm}$ $\mathrm{CaCl}_{2}, 0.4 \mathrm{mM} \mathrm{MgCl} 2,0.3 \mathrm{mg} / \mathrm{ml}$ penicillin, $0.1 \mathrm{mg} / \mathrm{ml}$ streptomycin, 50 $\mu \mathrm{M}$ leupeptin, $1 \mu \mathrm{M}$ aprotinin, $2 \mu \mathrm{M}$ pepstatin $\mathrm{A}, \mathrm{pH}$ 7.4), and centrifuged at $100,000 \times g$ for $30 \mathrm{~min}$. The concentration of membrane protein was measured according to Simpson and Sonne (1982). All procedures were performed at $4^{\circ} \mathrm{C}$ or on ice. Isolated membranes were stored at $-80^{\circ} \mathrm{C}$ until used.

Membranes were also prepared from cells of myogenic cell lines. These lines were generated by transformation of satellite cells from identified adult muscles, using retrovirus-mediated transfer of a temperature-sensitive oncogene (Donoghue et al., 1992a,b, 1996). Four cell lines were used: 6-3/A3, derived from the masseter muscle of the jaw; $2-1 / \mathrm{H}$, derived from the infrahyoid muscles of the neck; $5-7 / \mathrm{H}$ from tibialis anterior and extensor digitorum longus of the hindlimb; and 1-1/B1 from the gastrocnemius and plantaris of the hindlimb. In each case, cells were grown in serum-rich medium at $33^{\circ} \mathrm{C}$ for $7-10 \mathrm{~d}$, then transferred to serum-poor medium at $37^{\circ} \mathrm{C}$ to promote fusion into myotubes. Once fusion had occurred, cells were harvested and gently homogenized with a $5 \mathrm{ml}$ Dounce homogenizer in $4 \mathrm{vol}$ of homogenizing buffer for 130 strokes. The homogenate was then layered on a $5-50 \%$ sucrose gradient, and membranes were isolated as described above. Membranes from the two rostral lines were combined, as were membranes from the two caudal lines, to obtain sufficient material for assay.

Preparation of membrane lanes and carpets. Alternating membrane lanes (stripes) were prepared as described by Walter et al. (1987a). Rostral and caudal muscle membrane fragments were diluted with sterile PBS (in mM: $150 \mathrm{NaCl}, 13 \mathrm{Na}_{2} \mathrm{HPO}_{4}, 2.4 \mathrm{NaH}_{2} \mathrm{PO}_{4}$ ). The final concentration varied from 200 to $500 \mu \mathrm{g} / \mathrm{ml}$, but was always the same for rostral and caudal membranes within each experiment. Fluorescein- or rhodamine-labeled beads (Molecular Probes, Eugene, OR) were mixed with rostral or caudal membrane fragments, respectively, to visualize alternating lanes. A silicone matrix grid consisting of $90 \mu \mathrm{m}$ alternating lanes was used. A nucleopore filter (Corning, Cambridge, MA) was treated with $20 \mu \mathrm{g} / \mathrm{ml}$ laminin (Calbiochem) and placed onto this grid. A $100 \mu \mathrm{l}$ droplet of the rostral membrane suspension was then applied to the filter and sucked through using a vacuum unit (WPI Instruments) under -300 psi pressure. The filter, now bearing lanes of membrane (lane A) separated by membrane-free lanes, was transferred onto a second silicone matrix, consisting of a fine mesh, and a $100 \mu \mathrm{l}$ droplet of caudal membrane suspension was applied under vacuum $(-200 \mathrm{psi})$, forming lane B. The second solution of membranes adhered primarily to those regions of the filter not already occupied and formed a second lane, designated lane B (illustrated in Walter et al., 1987a). Any loosely bound membranes were removed by gently rinsing the filters in PBS, and the filters were transferred immediately into culture medium for co-culture. The order of applying rostral or caudal membranes to either lane A or B was alternated with each filter. Control experiments showed no consistent difference in results between lanes $\mathrm{A}$ and $\mathrm{B}$.

Preparation of embryonic spinal cord transverse slices. Rostral (cervical) and caudal (lumbar) spinal cords were isolated from E15 rat embryos and cut into $300 \mu \mathrm{m}$ transverse slices with a Vibratome (Xie and ZiskindConhaim, 1995). Spinal cord slices were placed on the membranebearing filter and incubated in six-well dishes in $5 \% \mathrm{CO}_{2}$ at $37^{\circ} \mathrm{C}$ for $3 \mathrm{~d}$. Culture medium was made according to $\mathrm{Xie}$ and Ziskind-Conhaim (1995) and consisted of (per $100 \mathrm{ml}) 50 \mathrm{ml}$ DMEM, $25 \mathrm{ml}$ HBSS, $15 \mathrm{ml}$ doubly distilled $\mathrm{H}_{2} \mathrm{O}, 2.3 \mathrm{ml} 20 \%$ glucose, $1.6 \mathrm{ml} 23.8 \%$ HEPES buffer, $\mathrm{pH} 7.2,8 \mathrm{ml}$ heat-inactivated fetal bovine serum, $400 \mu \mathrm{g}$ NGF, $5 \mu \mathrm{g}$ CNTF (Regeneron Pharmaceuticals), $0.03 \mathrm{~g}$ penicillin, and $0.01 \mathrm{~g}$ streptomycin.

Immunocytochemical staining of neurites. The method of Donoghue et al. (1996) was used to stain neurites growing from explants. Cultures were fixed with buffered $4 \%$ paraformaldehyde for $1 \mathrm{hr}$ at $4^{\circ} \mathrm{C}$. After a rinse in PBS, the cultures were treated for $20 \mathrm{~min}$ with blocking solution (4\% BSA, and $0.5 \%$ Triton X-100 in PBS). The cultures were incubated in anti-neurofilament (NF) (Sigma, St. Louis, MO; 1:100) overnight, rinsed in PBS, incubated for $1 \mathrm{hr}$ in fluorescein-labeled secondary antibody (Vector Laboratories, Burlingame, CA; diluted 1:100), washed again, and coated with one drop of Vectashield medium (Vector Laboratories). In some cases neurites were stained with biotinylated anti-NF (Vector Laboratories), followed by the HRP reaction in the Vector ABC kit (Donoghue et al., 1996). Some spinal cord neurites were immunostained for choline acetyltransferase (ChAT), following the method of Houser et al. (1983) and Phelps et al. (1990). After 3 d, co-cultures were fixed and blocked as described above. Cultures were then incubated in anti-ChAT, a monoclonal antibody from mouse hybrid cells (Boehringer Mannheim, Indianapolis, IN; 1:10 in the blocking buffer) for 2-3 hr at $37^{\circ} \mathrm{C}$. Cultures were next incubated in anti-mouse $\operatorname{IgG},(\gamma$-specific $)$ fluorescein-conjugated antibody (affinity-purified from goat; Boehringer Mannheim; 1:200) for $1 \mathrm{~h}$ at room temperature. Controls were incubated in secondary antibody without primary.

Estimates of selective neurite growth. Outgrowth on striped substrates was rated as being either selective or random. When neurites grew almost entirely on one lane type for the entire $3 \mathrm{~d}$ culture period, growth was rated as being selective. All other cultures were scored as random. This method of scoring is very likely to have underestimated the degree of selectivity we report, because many cultures that exhibited incomplete but significant preferences were scored as random. However, we chose this binary scoring system to exclude any possibility of subjective bias. To prevent bias in judging selectivity, each culture was photographed, and slides were projected on a screen. Observers then evaluated selectivity of growth without knowing the identity of the culture.

Only co-cultures that met the following criteria were scored: (1) lanes were distinct, as detected by fluorescein- or rhodamine-labeled beads; (2) axons were stained adequately to visualize their position on lanes; and (3) outgrowth was sufficient to judge degree of selectivity. Neurite length was determined from the average of three measurements of the distance from the edge of the ventral surface of the spinal cord slice to the leading edge of growing neurites. Data are presented as means \pm SEs and were analyzed by Student's $t$ test.

A relatively large proportion of co-cultures exhibited no selectivity. Much of the apparent nonselectivity is likely to reflect the strict criteria we used in accepting growth as selective. In addition, however, we noticed that growth patterns of neurites of all four spinal cord explants on each filter were similar. Thus, there is real variability that is unlikely to reflect the precise spinal level from which slices were taken, but rather appears to result from variations among substrates. These variations, in turn, could arise from biological differences among membrane preparations, instability of the recognition molecules, or technical variations in the efficacy with which filters were coated. At present we have no way of distinguishing among these explanations. 

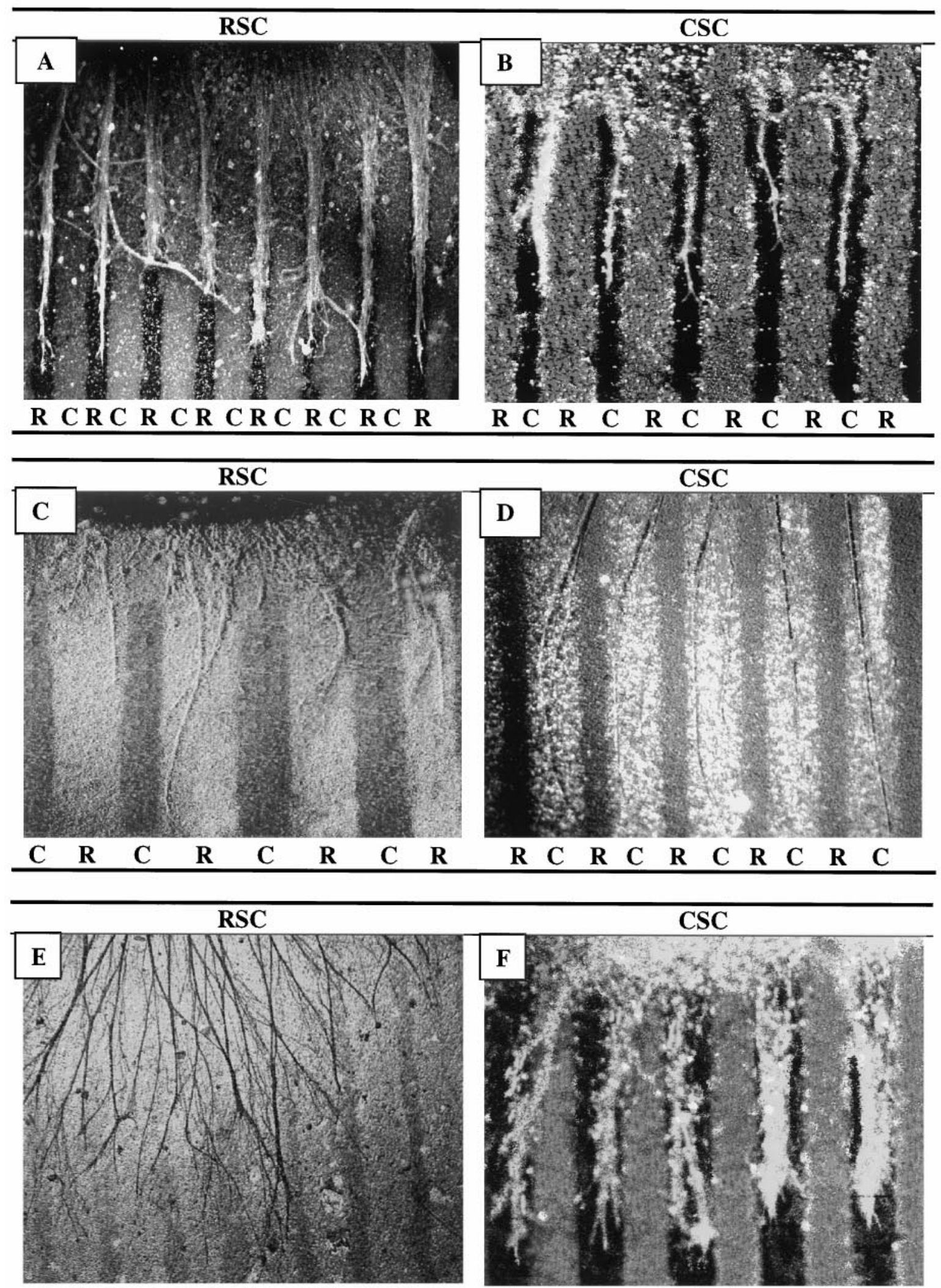

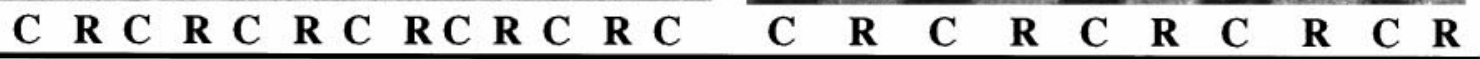

Figure 1. Position-dependent preferential outgrowth of spinal neurites on membranes from P0 and E18 limb muscles. Spinal cord slices cut from the cervical (rostral, $R S C$ ) or lumbar (caudal, $C S C$ ) enlargement were placed on alternating stripes of membrane derived from forelimb (rostral) or hindlimb (caudal) muscles. Membranes applied to one set of lanes in each culture were mixed with fluorescein-labeled beads to mark lane boundaries and marked rostral $(R)$ or caudal $(C)$. After $3 \mathrm{~d}$, the cultures were fixed, and neurites were labeled with antibodies to neurofilaments $(A, B)$, (Figure legend continues) 


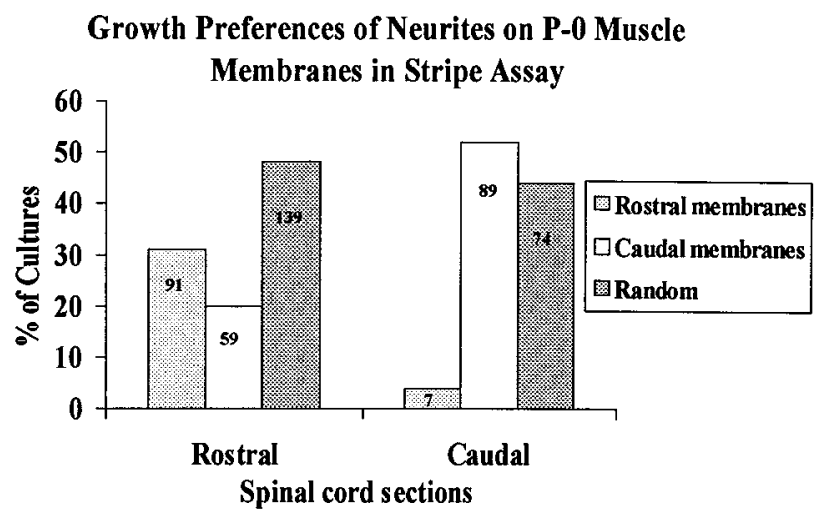

Figure 2. Position-dependent preferential outgrowth of spinal neurites on membranes from P0 limb muscles. Cultures were prepared as in Figure 1. Columns show percentage of cultures showing preferential outgrowth on rostral or caudal membranes or no striking preference (Random). Numbers indicate number of cultures in each category.

\section{RESULTS}

\section{Selective growth of neurites on membranes from forelimb and hindlimb muscle}

Our aim is to localize and characterize muscle-associated cues that mediate the topographically selective innervation of muscle.
We therefore began the present study by asking whether motor neurons can distinguish membranes from rostral and caudal muscles. We used explants of E15 spinal cord as a source of motor neurons for three reasons. First, neurite outgrowth is robust at this stage. Second, nearly all of the neurites that leave such explants are derived from motor neurons (Xie and ZiskindConhaim, 1995). Third, intramuscular topography is detectable in vivo by E15. On the other hand, it was not feasible to obtain sufficient amounts of material for membrane preparation from individual muscles or muscle groups at this stage, so we used muscles from E18 and neonates (P0).

In the first experiment, membranes from $\mathrm{P} 0$ forelimb (rostral) and hindlimb (caudal) muscles were arrayed in alternating lanes on a nucleopore membrane, using a suction apparatus (Walter et al., 1987a). Fluorescent microspheres were mixed with one of the two membrane preparations to facilitate subsequent visualization of lane borders and to confirm that mixing of membrane from the two sources was minimal. Slices of spinal cord from the cervical or lumbar enlargement (called rostral and caudal, respectively, here) were placed atop the membranes, so neurites had equal access to both types of lanes.

Two cultures from this series are illustrated in Figure $1 A, B$, and results from all 459 cultures in this series are summarized in Figure 2. In approximately one-half (89/170) of the cultures made

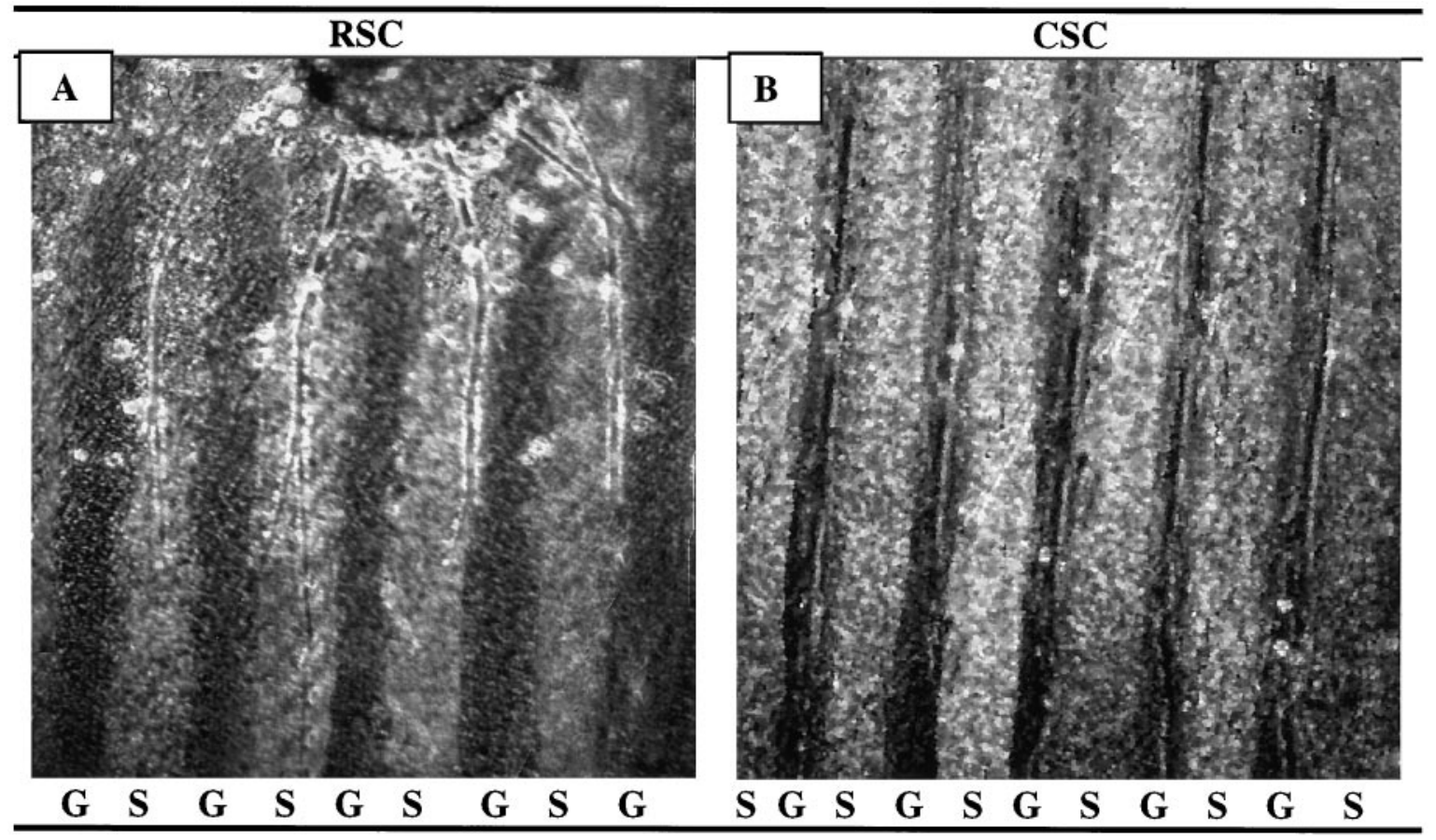

Figure 3. Position-dependent preferential outgrowth of spinal neurites on membranes from individual muscles. Spinal cord slices cut from the cervical (rostral, $R S C$ ) or lumbar (caudal, $C S C$ ) enlargement were placed on alternating stripes of membrane derived from the serratus anterior ( $S$ ), a rostral muscle, or from the gluteus $(G)$, a caudal muscle. Membranes applied to one set of lanes in each culture were mixed with fluorescent beads to mark lane boundaries. After $3 \mathrm{~d}$, the cultures were fixed, and neurites were labeled with antibodies to neurofilaments. (Figures shown are unstained.) $A$, Rostral spinal cord neurites grew selectively on serratus anterior membranes in $36 \%$ of cultures. $B$, Caudal neurites grew selectively on gluteus membranes in $47 \%$ of cultures. Magnification: $A, 100 \times ; B, 80 \times$.

left unstained $(C, D)$, or reacted with HRP-DAB $(E)$ (see Materials and Methods). Rostral spinal cord neurites grew selectively on P0 rostral membranes in $31 \%$ of cultures $(A)$ and on E18 rostral membranes in $9 \%$ of cultures $(C)$. Caudal spinal cord neurites grew selectively on caudal P0 membranes in $52 \%$ of cultures $(B)$ and on caudal E18 membranes in $78 \%$ of cultures $(D)$. E, Rostral neurites grew randomly in $42 \%$ of cultures on E18 rostral membranes. $F$, Neurites of caudal spinal cord growing on caudal P0 membranes and stained with fluorescein-labeled antibody to acetylcholine transferase. Magnification: $A, E, 60 \times ; B, D, F, 100 \times ; C, 140 \times$. 


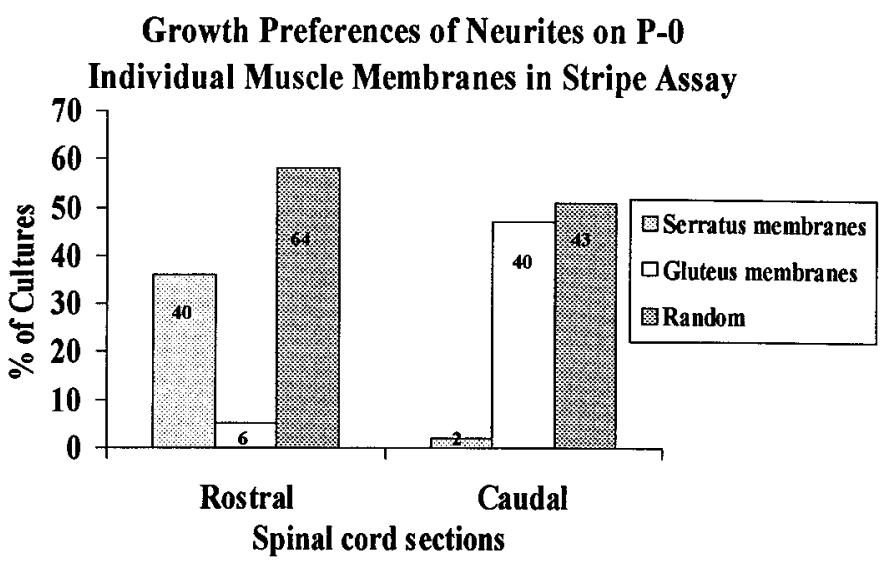

Figure 4. Position-dependent preferential outgrowth of spinal neurites on membranes from individual muscles. Cultures were prepared as in Figure 3. Columns show percentage of cultures showing preferential outgrowth on rostral (serratus anterior) or caudal (gluteus) membranes or no striking preference (Random). Numbers indicate number of cultures in each category.

with caudal spinal cord slices, neurites showed a striking preference for caudally derived membranes (Fig. $1 B$ ). In most of the remaining caudal spinal cord cultures (74/170), neurites crossed between lanes and showed no striking preference; rostral membranes were the preferred substrate in $<5 \%$ of cultures $(7 / 170)$. Thus, caudally derived motor neurites prefer caudal to rostral muscle membranes.

The behavior of neurites from rostral spinal cord was different from that of caudal neurites. Rostral neurites preferred rostrally derived muscle membranes in $31 \%$ (91/289) of the cultures (Fig. $1 A)$, preferred caudally derived membranes in $20 \%(59 / 289)$ of the cultures, and showed no clear preference in approximately one-half of the cultures (139/289). Thus, preferences of rostral neurites were biased toward rostrally derived muscle membranes. These preferences were not, however, precisely complementary to those of caudal neurites. Neurites from a significant minority of rostral explants grew preferentially on caudally derived muscle membranes, whereas neurites from very few caudal explants grew preferentially on rostrally derived membranes.

\section{Selective growth of neurites on membranes from serratus and gluteus muscles}

We tested two possible explanations for the incomplete preference of rostral and caudal neurites for muscles from corresponding axial levels. First, if expression of muscle-associated molecules that promote selective outgrowth were developmentally regulated, their levels might have declined greatly from peak values by P0. Therefore, we assessed the outgrowth of neurites from rostral and caudal spinal cord on membranes from forelimb and hindlimb muscles of E18 embryos (P0 being equivalent to E22) (Fig. $1 C, D)$. In this set of cultures, preferences were similar to those documented above for P0 muscle membranes: neurites from caudal spinal cord explants $(n=46)$ extended preferentially on caudal membranes in most cultures (78\%), grew randomly in the remainder, and extended preferentially on rostral membranes in none. In contrast, neurites from rostral spinal cord extended preferentially on rostral membranes in a minority of cultures (7/78 or $9 \%$ ) (Fig. 1C) and either extended preferentially on

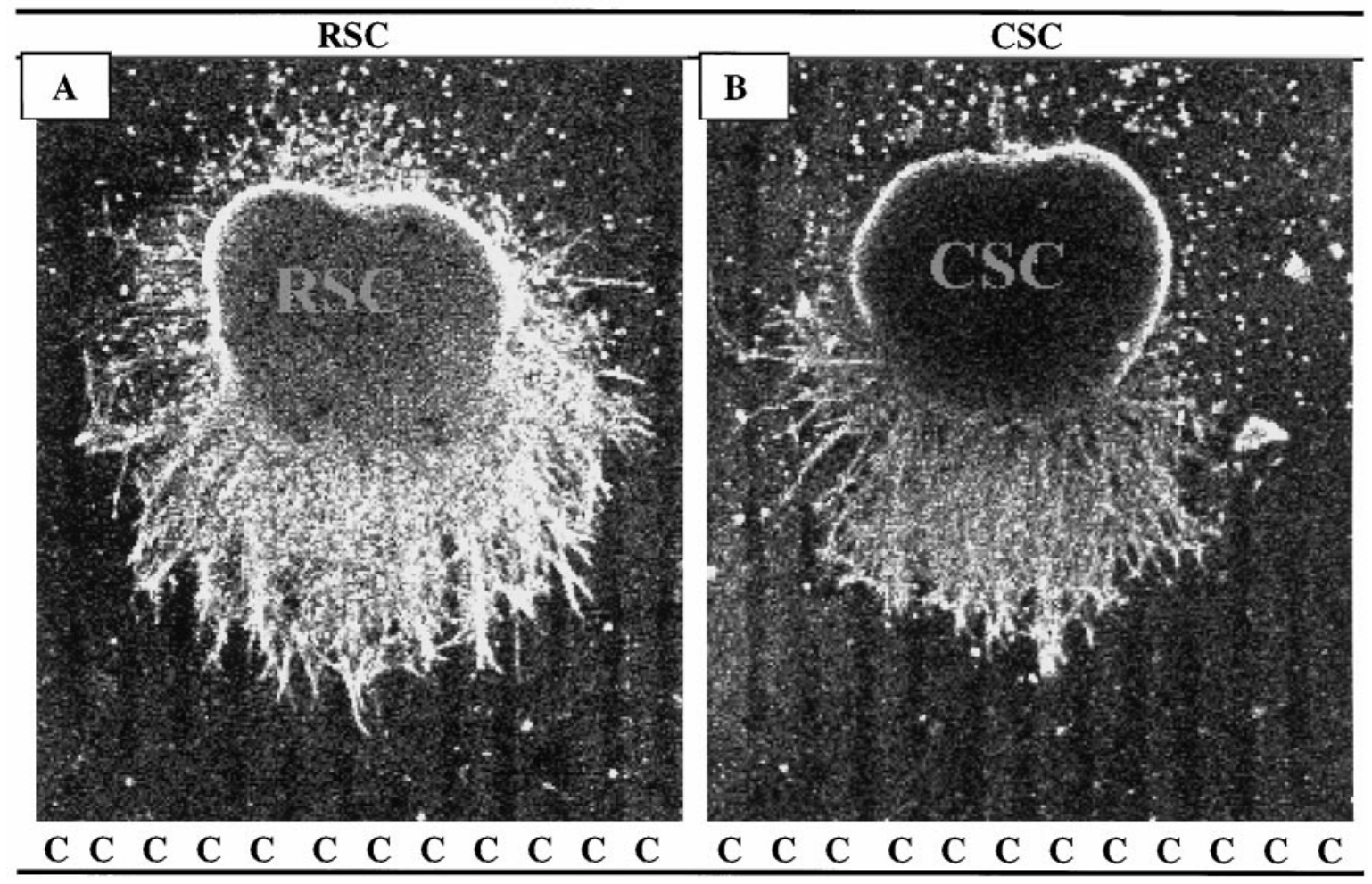

Figure 5. Rostral spinal cord neurites grew longer than caudal neurites on membrane lanes. Spinal cord slices cut from the cervical (RSC) or lumbar (CSC) enlargement were placed on stripes of membrane derived from hindlimb ( $C$, caudal) muscles (ventral surface of slice is down). Membranes mixed with fluorescent beads were applied to one set of lanes in each culture; intervening dark lanes were uncoated. After $3 \mathrm{~d}$, the cultures were fixed, and neurites were labeled with antibodies to neurofilaments. $A$, Rostral neurites growing on caudal membranes. $B$, Caudal neurites growing on caudal membranes. Both $A$ and $B$ are at $40 \times$ magnification. 
caudal membranes (49\%) or grew randomly (42\%) in the remainder (Fig. 1E). Thus, the ability of muscle membranes to promote positionally selective outgrowth does not decline dramatically between E18 and P0.

Second, we considered the possibility that forelimb and hindlimb each contained muscles that differed in their abilities to promote selective outgrowth, with the overall preference reflecting some average of individuals in the mixture. Accordingly, we prepared and tested membranes from individual muscles at P0. We chose serratus anterior and gluteus maximus as representative rostral and caudal muscles, respectively, because motor axons form topographic maps within both (Brown and Booth, 1983a,b; Laskowski and Sanes, 1987). Results from this series of 195 cultures are shown in Figures 3 and 4. Indeed, positional preferences of neurites were more striking when their choice was between membranes from serratus anterior and gluteus maximus than when they were forced to choose between membranes from mixtures of forelimb and hindlimb muscles. Thirty-six percent of rostral neurites and $47 \%$ of caudal neurites selectively extended neurites on membranes from a corresponding axial level. Most of the other half failed to exhibit clear preferences, and very few grew selectively on positionally mismatched membranes $(6 / 110$ rostral and 2/85 caudal explants). Thus spinal cord neurites can differentiate between positional cues expressed by individual muscles.

\section{Growth of neurites on uniform substrates}

Selective outgrowth of neurites on positionally matched membranes implies that neurites from one level prefer membranes from the corresponding level. This preference could have any number of cellular explanations. Perhaps the simplest possibility is that membranes are more potent promoters of neurite outgrowth from a similar axial level. To test this possibility, we grew rostral and caudal spinal cord explants on stripes of membranes from a single level. The membranes were applied to nitrocellulose filters in stripes, with intervening lanes uncoated, to orient neurite outgrowth.

Figure 5 shows examples from this series, and Figure 6 summarizes the results. On average, neurites from rostral spinal cord explants were longer than those from caudal explants on either rostrally or caudally derived muscle membranes. This difference might reflect a general difference between the health of neurons in rostral and caudal explants. To test this possibility, we compared the growth of rostral and caudal explants on laminin, a promoter of neurite outgrowth from most neuronal types (Sanes, 1989). In such comparisons, there was no statistically significant difference in average length between rostral and caudal neurites (678 $\pm 29 \mu \mathrm{m}$ vs $624 \pm 31 \mu \mathrm{m})$. In addition, it is noteworthy that the average extent of outgrowth on laminin was similar to that on muscle membranes, suggesting that the membranes do contain potent promoters of neurite outgrowth.

\section{Position-dependent choices between muscle membranes and laminin}

Outgrowth of neurites from either rostral or caudal spinal cord explants is promoted to a similar extent by uniform substrates of either rostrally or caudally derived muscle membranes (Fig. 6). Yet neurites can distinguish rostral from caudal membranes when both are present (Figs. 1-4). Together, these results suggest that neurites compare rostral with caudal membranes and make directional choices depending on the outcome of the comparison. An important question is whether this hypothetical computation

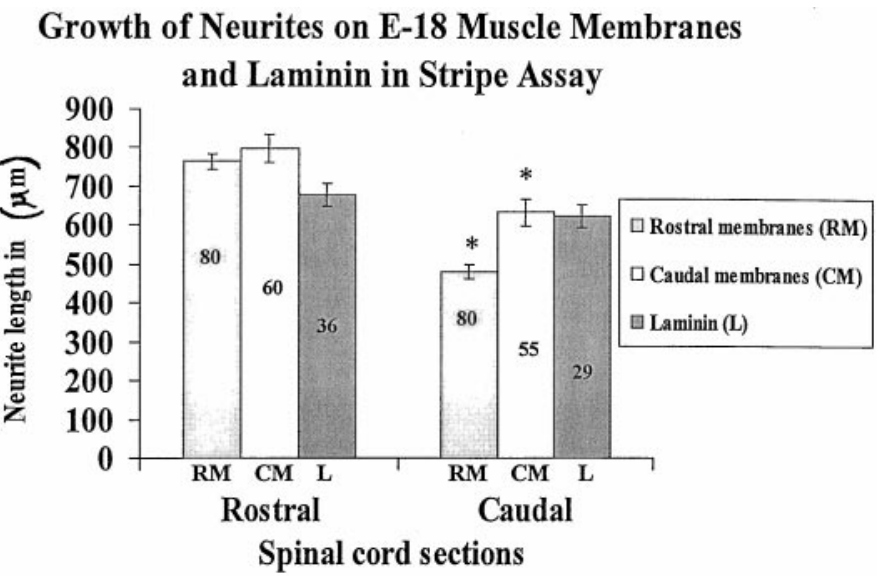

Figure 6. Spinal cord neurites grow similar lengths on rostral and caudal membranes and on laminin. Cultures were prepared as in Figure 5. Membranes or laminin were applied to one set of lanes in each culture. Intervening lanes were uncoated. Measurements of neurite length were made in cultures as in Figure 5. Columns show average neurite length $( \pm \mathrm{SE})$, measured as the distance from the edge of the explant to the end of the longest neurite. Extent of outgrowth on laminin-coated stripes (data not shown, but see Figure 7) is given for comparison. Numbers indicate number of cultures in each category. Asterisks indicate that caudal neurites grew significantly less than rostral neurites on both rostral and caudal membranes $(p<0.05)$.

requires a direct comparison between membranes from rostral and caudal muscles or whether there is a general hierarchy of preferences that guide axonal behavior.

To distinguish between these alternatives, we compared rostrally and caudally derived membranes separately with laminin (Sanes, 1989). Examples are shown in Figures 7, and results are tabulated in Figure 8. As in the assays described above, neurites crossed boundaries between lanes in many cultures (see Fig. 8 legend). In those cultures that did show outgrowth confined to lanes, however, positional preferences were clear. Rostral neurites preferred rostrally derived membranes over laminin (selective growth on 33 vs 12 cultures, respectively). Rostral neurites also preferred laminin to caudally derived membranes (13 vs 2 cultures). Conversely, caudal neurites preferred caudally derived membranes to laminin (46 vs 5 cultures), and caudal neurites preferred laminin to rostral membranes (58 vs 28 cultures). Thus, the preferred order of growth for rostral neurites is rostrally derived membranes $>$ laminin $>$ caudally derived membranes, whereas the preferred order of growth for caudal neurites is caudally derived membranes $>$ laminin $>$ rostrally derived membranes. These results show that growing neurites respond to a choice in substrate and have clear preferences for positionspecific membranes.

\section{Selective growth of neurites on membranes from cultured myotubes}

Membrane preparations from embryonic and neonatal muscles are predominantly derived from myotubes but also contain contributions from non-muscle cells such as axons, Schwann cells, vascular endothelial cells, and fibroblasts. Any of these cells could be sources of guidance information for motor axons. To determine whether recognition molecules are present in myotube membranes, we used muscle cell lines derived from identified muscles (Donoghue et al., 1992a). These lines were generated by immortalizing satellite cells with a temperature-sensitive oncogene. The cells grow as myoblasts in serum-rich medium at $33^{\circ} \mathrm{C}$, 

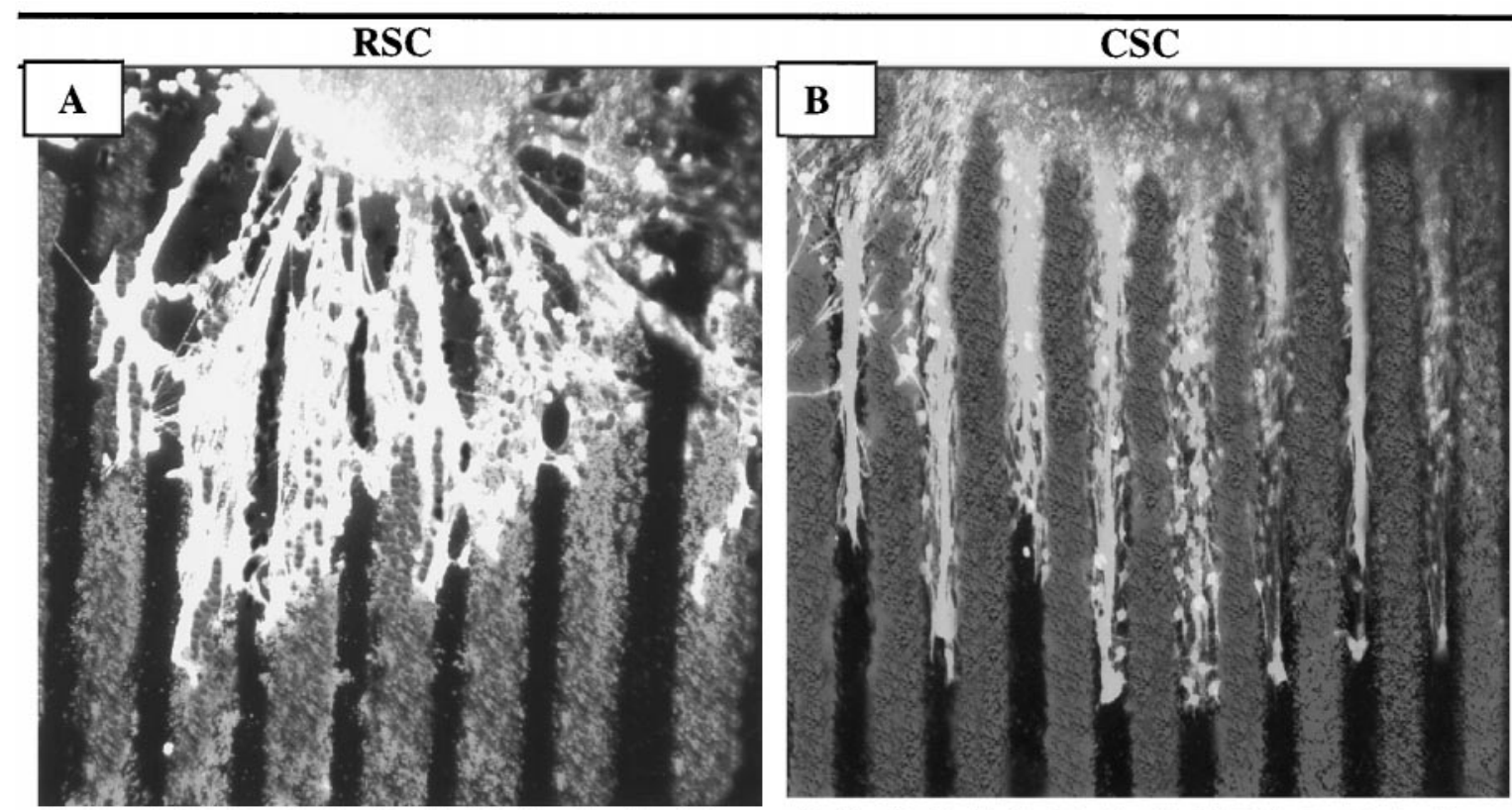

L R L R L R L R L R L R L R

R L R L R L R L RLRLR L RL

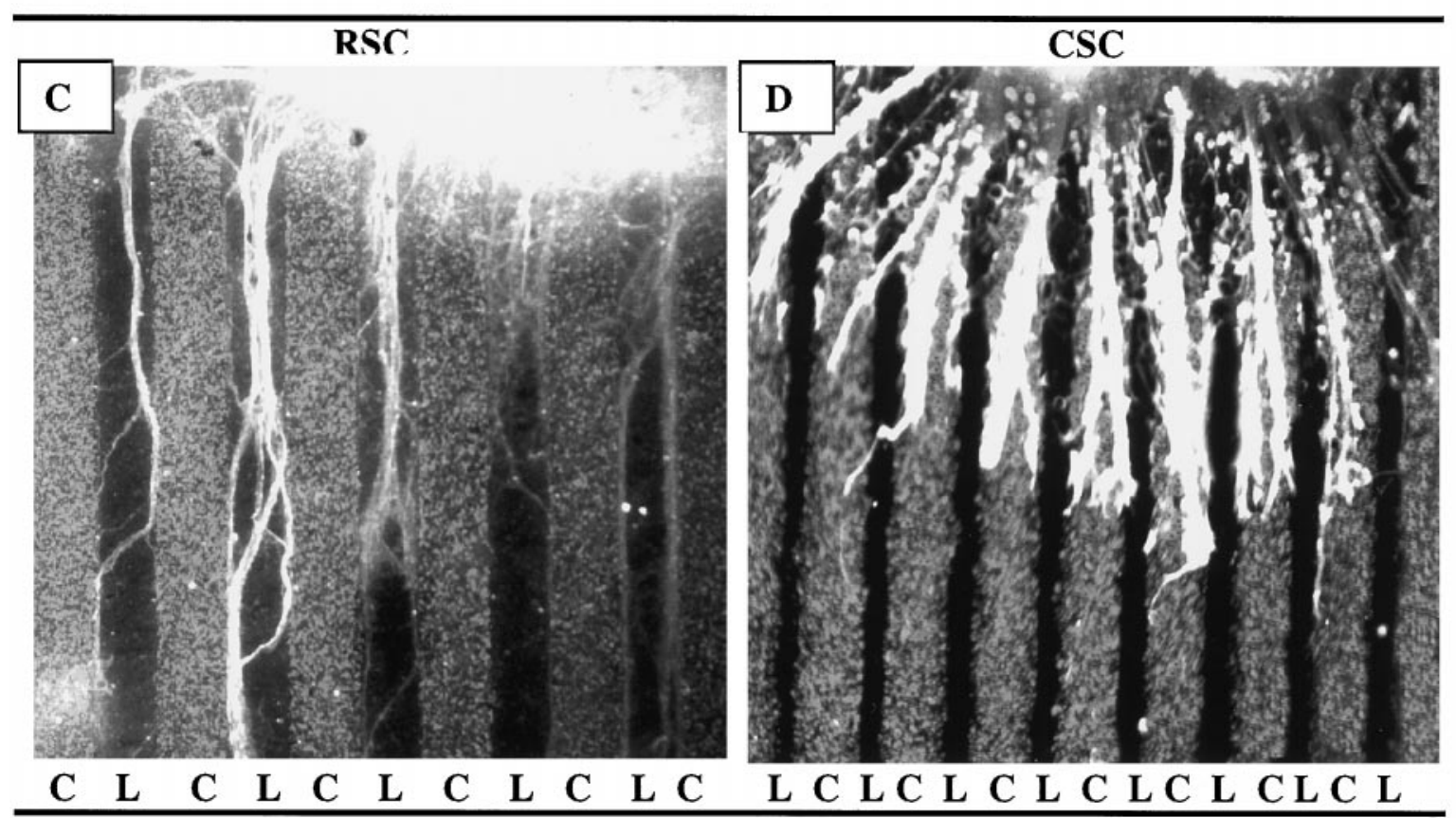

Figure 7. Spinal cord neurites make position-dependent choices between muscle membranes and laminin. Spinal cord slices cut from the cervical (rostral, $R S C$ ) or lumbar (caudal, $C S C$ ) enlargement were placed on alternating stripes of muscle membranes $(R$, rostral, or $C$, caudal) and laminin. $A$, Rostral spinal cord neurites prefer rostral membranes to laminin. $B$, Caudal neurites prefer laminin to rostral membranes. $C$, Rostral neurites prefer laminin to caudal membranes. $D$, Caudal neurites prefer caudal membranes to laminin. Neurites were stained with fluorescein-anti-NF. Magnification: $A, 80 \times ; B, D, 60 \times ; C, 100 \times$.

then fuse to form myotubes when switched to a serum-poor medium at $37^{\circ} \mathrm{C}$. The lines were generated from mice bearing a transgene expressed in a rostrocaudal gradient in axial muscles (Donoghue et al., 1991), and these lines express the transgene at levels that correspond to the axial position from which they were derived. Thus, muscles bear a cell-autonomous, heritable memory of their axial position, and this memory is retained by the immortalized cell lines (Donoghue et al., 1992a,b).

In the present experiments, we prepared membranes from myotubes of lines derived from the masseter muscle of the jaw, the infrahyoid muscles of the neck, and crural muscles (tibialis anterior, gastrocnemius, extensor digitorum longus, and plantaris) of the hindlimb. To obtain sufficient material for assays, membranes from two rostrally derived lines were mixed, as were membranes from two caudally derived lines. Spinal cord explants were then cultured as described above on alternating lanes of membranes from the rostral and caudal cell lines.

Neurites exhibited preferences between the cell lines that cor- 


\section{Growth Preferences of Neurites on P-0 Muscle Membranes vs Laminin in Stripe Assay}

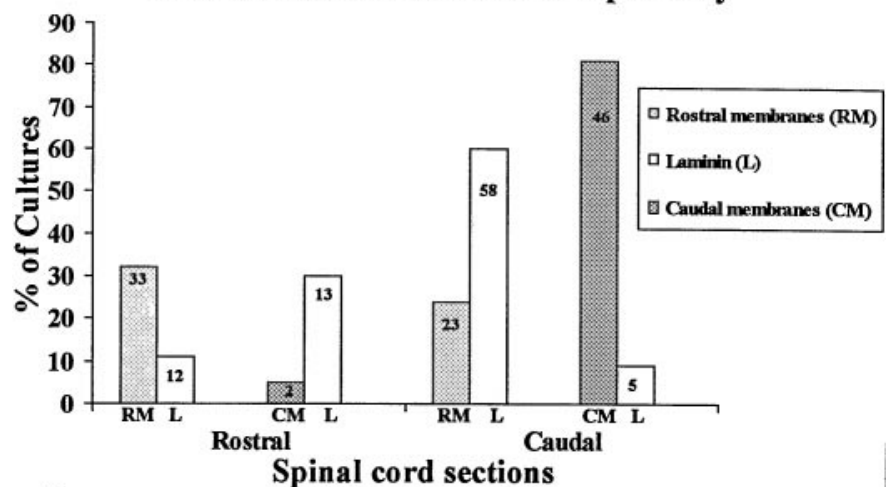

Figure 8. Spinal cord neurites make position-dependent choices between muscle membranes and laminin. Cultures were prepared as in Figure 7, in which alternating lanes contained rostral membranes, caudal membranes, or laminin. Columns show percentage of cultures showing preferential outgrowth on rostral or caudal membranes $(R M$ or $C M)$ or laminin $(L)$. Numbers indicate number of cultures in each category. An additional 108 cultures, not included in this graph, did not show striking preferences for either membranes or laminin $(59,28,15$, and 6 in cocultures of the types shown in Figure $7 A-D$, respectively).

responded closely to their preferences between rostral and caudal neonatal muscles (Figs. 9, 10; compare with Figs. 1-4). Neurites from caudal spinal cord grew selectively on membranes from the caudally derived cell lines in three-fourths of the assays and exhibited no clear preference in the remainder; in no case were membranes from rostrally derived cell lines preferred. In contrast, neurites from rostral spinal cord grew selectively on membranes from rostrally and caudally derived cell lines in approximately equal fractions $(\sim 40 \%)$ of the cultures. Thus, myotubes contain cues sufficient to account for several axonal behaviors seen on crude muscle membranes.

\section{DISCUSSION}

The most intensively studied topographic map of neural connectivity is that formed by retinal axons on the optic tectum (Sperry, 1963). A crucial step in elucidating mechanisms that underlie formation of the map was the introduction of the membrane stripe assay by Bonhoeffer and his colleagues. In their initial studies, they used it to assay growth of retinal axons on membranes derived from rostral and caudal tectum (Walter et al., 1987a,b). By offering the axons a choice between substrates, they were able to detect outgrowth-inhibiting as well as outgrowthpromoting cues and to detect axonal behaviors that would have escaped detection on uniform substrates. Their results provided a starting point for molecular investigations that recently culminated in the demonstration that the Eph kinases and their ligands, the ephrins, play critical roles in the formation of the map (for review, see Flanagan and Vanderhaeghen, 1998).

In previous studies, we and others have shown that motor axons form orderly position-dependent maps of connectivity with skeletal muscles (see introductory remarks). In view of the parallels between visuotopic and neuromuscular maps, we adapted the stripe assay to investigate the preferences of motor axons for muscle membranes. We used explants of spinal cord as a source of motor neurons, because essentially only motor axons grow out of such explants in the absence of specific tropic factors such as netrins. We provided the neurites choices between (1) membranes from groups of hindlimb and forelimb muscles, (2) membranes from serratus anterior and gluteus muscles, and (3) membranes from head- and hindlimb muscle-derived cell lines. We also assayed growth on substrates that gave neurites a choice between a strong growth-promoting substrate, laminin, and mem-

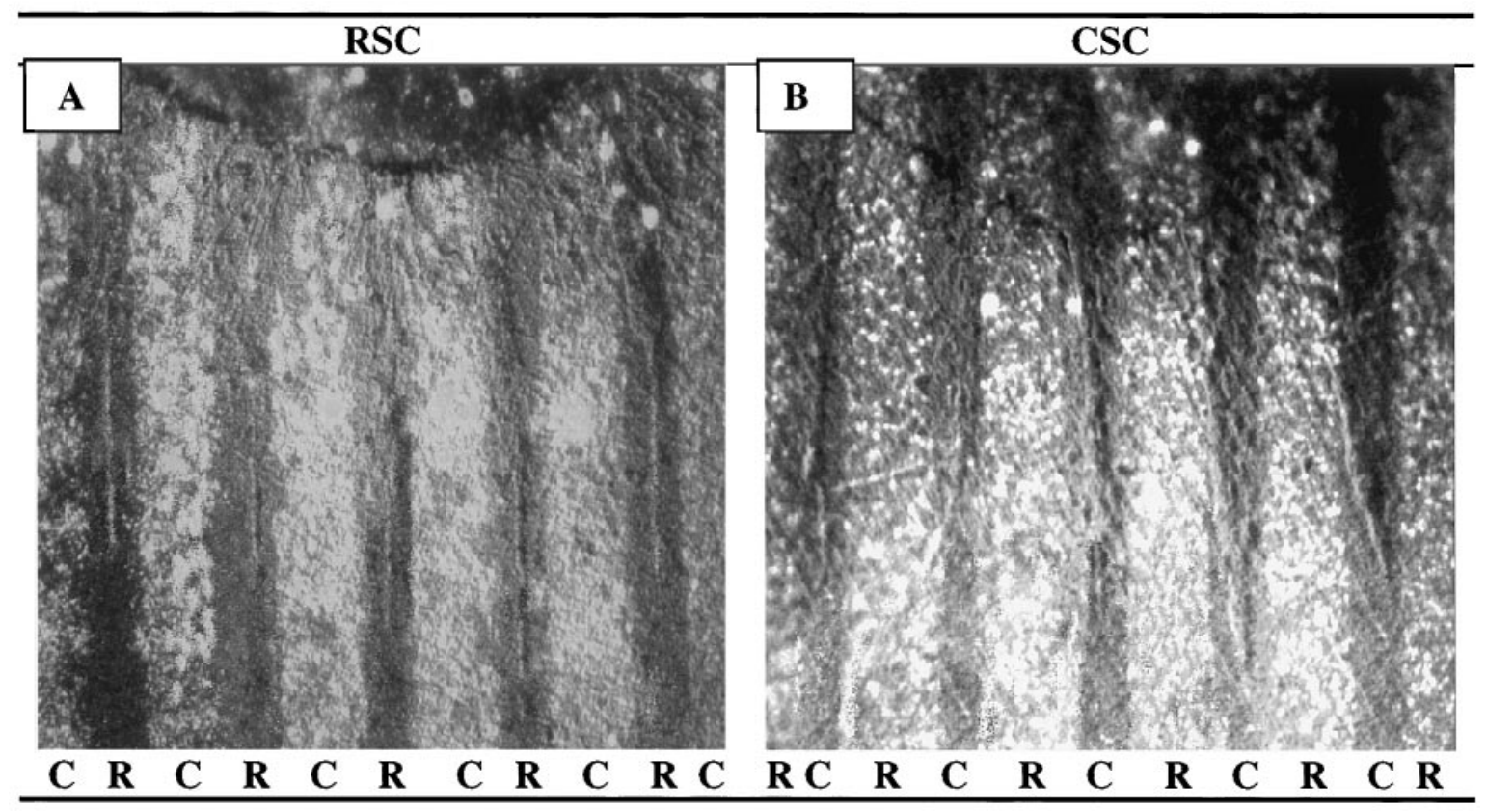

Figure 9. Position-dependent preferential outgrowth of spinal neurites on membranes from muscle cell lines. Spinal cord slices cut from the cervical (rostral, $R S C$ ) or lumbar (caudal, CSC) enlargement were placed on alternating stripes of myotube membranes prepared from immortalized cell lines. Membranes applied to one set of lanes in each culture were mixed with fluorescent beads to mark lane boundaries ( $R$, rostral; $C$, caudal). After $3 \mathrm{~d}$, the cultures were fixed, and neurites were labeled with fluorescein-labeled antibodies to neurofilaments. $A$, Rostral spinal cord neurites grew selectively on membranes from the rostrally derived cell lines. $B$, Caudal neurites grew selectively on membranes from the caudally derived cell lines. Magnification: $100 \times$. 


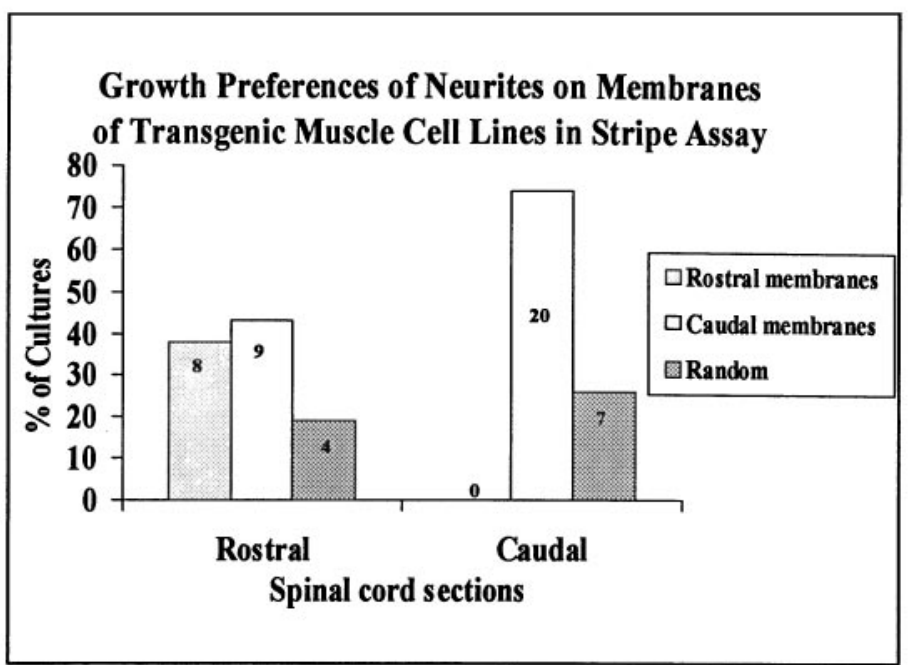

Figure 10. Position-dependent preferential outgrowth of spinal neurites on membranes from muscle cell lines. Cultures were prepared as in Figure 9. Columns show percentage of cultures showing preferential outgrowth on rostral or caudal membranes or no striking preference (Random). Numbers indicate number of cultures in each category.

branes from hindlimb or forelimb muscles. Neurites showed clear preferences in each of these four situations. Moreover, the neuritic preferences differed in a systematic way depending on whether the spinal cord explant was derived from cervical (rostral) or lumbar (caudal) levels. Most strikingly, caudally derived neurites preferred caudal muscle membranes to rostral muscle membranes or laminin. In contrast, rostrally derived neurites either grew selectively on rostrally derived membranes or exhibited little positional preference. Together, our results provide evidence that (1) myotube membranes bear guidance cues whose nature or amount varies with their rostrocaudal position of origin, and (2) embryonic motor axons recognize these cues by means of receptors whose nature or amount varies with spinal position.

These results lead to a series of new questions about the nature of neuromuscular positional cues. First, how do the axonal receptors vary with position? Here, our comparison was limited to explants from the cervical and lumbar enlargements. The behaviors we observed might represent two samples from a gradient of preferences, but they might also represent specific behaviors of motor neurons that innervate forelimb and hindlimb muscles. A simple way to distinguish these alternatives is to assay additional spinal levels. If preferences are graded, explants from intermediate positions are predicted to show intermediate preferences, and explants from more rostral or caudal positions may show more extreme preferences. It will also be important to determine whether all motor neurons at each level express the same preference, or whether axonal choices vary among or within motor pools. Unfortunately, however, more complex assays will be needed to answer this question. Despite recent progress in identification of transcription factors that mark distinct motor pools (Tsuchida et al., 1994; Sockanathan and Jessell, 1998), no axonal markers are currently available. One consistent feature of our results is that caudally derived neurites appear to be more sensitive than rostral neurites to positional differences among muscle membranes. Some of this difference may reflect the advanced maturity of the rostral cord at any given chronological age. For example, receptors on spinal neurites may be expressed during a limited, early period in development. Alternatively, our assay may be better suited for detecting those differences to which caudal neurites respond. In fact, this explanation was originally advanced to account for the fact that temporal but not nasal axons recognized differences between rostral and caudal tectal membranes in the stripe assays of Bonhoeffer and colleagues (Walter et al., 1987a,b).

Second, we do not know, however, whether such cues are continuously graded in type or amount along the rostrocaudal axis. To address such issues, it will be important to assay additional muscles and also to modify the assay to permit investigation of "multiple choice" behavior. Moreover, we assayed differences between muscles that are never alternative choices of a single motor axon in vivo. Physiological data indicate that motor axons make intramuscular choices, forming maps within individual muscles. These cases are particularly revealing, because they most clearly involve target recognition and synaptic choices. We speculate that the intermuscular differences detected in the present study are related to the intramuscular differences that axons normally detect. However, we have no direct evidence on this point. It will therefore be important to modify or miniaturize the assay to investigate intramuscular differences.

Third, are axonal preferences manifested as preferential outgrowth important for synaptogenesis? In the retinotectal system, stripe assays are appropriate in that the phenomenon in vivo appears to involve guidance of growing axons. In muscle, in contrast, there appears to be a positional bias in synapse formation (Laskowski and High, 1989; Laskowski and Owens, 1994). It is tempting to speculate that the same cellular interactions could lead to preferential outgrowth in vitro and preferential synaptogenesis in vivo. However, additional assays that measure synapse formation directly will be needed to test this idea.

Finally, what is the molecular basis for the positionally selective outgrowth we have measured? Attractive candidates include the Eph kinases and their ligands, the ephrins (Flanagan and Vanderhaegen, 1998). The EphA family of kinases and their cognate ligands, the ephrin As, have been shown to be important in establishing the map in the tectum (see introductory remarks), and recent observations favor the possibility that these molecules also contribute to the formation of the neuromuscular map. Two EphA kinases, EphA3 and EphA4, are selectively expressed by subsets of motor neurons (Kilpatrick et al., 1996; Ohta et al., 1996). One ligand for these receptors, ephrin A5, is expressed at higher levels by rostral muscles and muscle cell lines than by caudal muscles and muscle cell lines (Donoghue et al., 1996). Both ephrinA2 and ephrinA5 inhibit outgrowth of motor axons, and the inhibition is position dependent, with caudal motor neurons showing greater inhibition than rostral motor neurons (Donoghue et al., 1996; Ohta et al., 1996). On the basis of these observations, we have sought and now have obtained preliminary evidence that overexpression of ephrinA5 in muscles of transgenic mice alters intramuscular topographic mapping (Laskowski et al., 1998b).

Ephrins are unlikely to account for all of the nerve-muscle preferences detected either in vivo or in vitro, however, because these ligands are not distributed in a rostrocaudal gradient. Nonetheless, it is tempting to speculate that Eph kinases and ephrins form part of a molecular code that biases synapse formation in favor of positionally matched partners. The assay system described here will be useful in testing this idea, as well as in identifying the other components of the system that almost certainly exist. 


\section{REFERENCES}

Bennett MR, Lavidis NA (1984a) Segmental motor projections to rat muscles during the loss of polyneuronal innervation. Dev Brain Res 13:1-7.

Bennett MR, Lavidis NA (1984b) Development of the topographical projection of motor neurons to a rat muscle accompanies loss of polyneuronal innervation. J Neurosci 4:2204-2212.

Brown MC, Booth CM (1983a) Segregation of motor nerves on a segmental basis during synapse elimination in neonatal muscles. Brain Res 273:188-190.

Brown MC, Booth CM (1983b) Postnatal development of the adult pattern of motor axon distribution in rat muscles. Nature 304:741-742.

Brown MC, Hardman VJ (1987) A reassessment of the accuracy of reinnervation by motoneurons following crushing or freezing of the sciatic or lumbar spinal nerves of rats. Brain 110:695-705.

Cox EC, Muller B, Bonhoeffer F (1990) Axonal guidance in the chick visual system: posterior tectal membranes induce collapse of growth cones from the temporal retina. Neuron 2:31-37.

DeSantis M, Berger PK, Laskowski MB, Norton AS (1992) Regeneration by skeletomotor axons in neonatal rats is topographically selective at an early stage of reinnervation. Exp Neurol 116:229-239.

Donoghue MJ, Merlie JP, Rosenthal N, Sanes JR (1991) Rostrocaudal gradient of transgene expression in adult skeletal muscle. Proc Natl Acad Sci USA 88:5847-5851.

Donoghue MJ, Patton BL, Sanes JR, Merlie JP (1992a) An axial gradient of transgene methylation in murine skeletal muscle: genomic imprint of rostrocaudal position. Development 116:1101-1112.

Donoghue MJ, Morris-Valero R, Johnson YR, Merlie JP, Sanes JR (1992b) Mammalian muscle cells bear a cell-autonomous heritable memory of their rostrocaudal position. Cell 69:67-77.

Donoghue MJ, Lewis RM, Merlie JP, Sanes JR (1996) The Eph kinase ligand AL-1 is expressed by rostral muscles and inhibits outgrowth from caudal neurons. Mol Cell Neurosci 8:1-16.

Drescher U, Kremoser C, Handwerker C, Loschinger J, Noda M, Bonhoeffer F (1995) In vitro guidance of retinal ganglion cell axons by RAGS, a $25 \mathrm{kDa}$ tectal protein related to ligands for Eph receptor tyrosine kinases. Cell 82:359-370.

Flanagan John G, Vanderhaeghen P (1998) The ephrins and Eph receptors in neural development. Annu Rev Neurosci 21:309-345.

Friedman GC, O'Leary DDM (1996) Retroviral misexpression of engrailed genes in the chick optic tectum perturbs the topographic targeting of retinal axons. J Neurosci 16:5498-5509.

Frisen J, Yates PA, McLaughlin T, Friedman GC, O'Leary DMM, Barbacid M (1998) Ephrin-A5 (AL-1/RAGS) is essential for proper retinal axon guidance and topographical mapping in the mammalian visual system. Neuron 20:235-243.

Grow WA, Kendall-Wassmuth E, Ulibarri C, Laskowski MB (1995) Differential delay of reinnervating axons alters specificity in the rat serratus anterior muscle. J Neurobiol 26:553-562.

Hardman VJ, Brown MC (1987) Accuracy of reinnervation of rat internal intercostal muscles by their own segmental nerves. J Neurosci 7:1031-1036.

Houser CR, Crawford GD, Barber RP, Salvaterra PM, Vaughn JE (1983) Organization and morphological characteristics of cholinergic neurons: an immunocytochemical study with a monoclonal antibody to choline acetyltransferase. Brain Res 266:97-119.

Kilpatrick TJ, Brown A, Lai C, Gassmann M, Goulding M, Lemke G (1996) Expression of the Tyro 4/Mek 4/Cek 4 gene specifically marks a subset of embryonic motor neurons and their muscle targets. Mol Cell Neurosci 7:62-74.

Laskowski MB, High JA (1989) Expression of nerve-muscle topography during development. J Neurosci 9:175-182.

Laskowski MB, Owens JL (1994) Embryonic expression of motoneuron topography in the rat diaphragm muscle. Dev Biol 166:502-508.

Laskowski MB, Sanes JR (1987) Topographic mapping of motor pools onto skeletal muscles. J Neurosci 7:252-260.

Laskowski MB, Sanes JR (1988) Topographically selective reinnervation of adult mammalian skeletal muscles. J Neurosci 8:3094-3099.

Laskowski MB, Coleman H, Nelson C, Lichtman JW (1998a) Synaptic competition during the reformation of a neuromuscular map. J Neurosci 18:7328-7335.

Laskowski MB, Feng G, Nichol M, Sanes JR (1998b) Overexpression of ephrin A5 disrupts neuromuscular topography. Soc Neurosci Abstr 24:791.

Ohta K, Nakamura M, Hirokawa K, Tanaka S, Iwana A, Suda T, Ando M, Tanaka H (1996) The receptor tyrosine kinase, Cek 8, is transiently expressed on subtypes of motoneurons in the spinal cord during development. Mech Dev 54:59-69.

Phelps PE, Barber RP, Brennan LA, Maines VM, Salvaterra PM, Vaughn JE (1990) Embryonic development of four different subsets of cholinergic neurons in rat cervical spinal cord. J Comp Neurol 291:9-26.

Sanes JR (1989) Extracellular matrix molecules that influence neural development. Rev Neurosci 12:491-516.

Sanes JR (1993) Topographic maps and molecular gradients. Curr Opin Neurobiol 3:67-74.

Simpson IA, Sonne O (1982) A simple, rapid and sensitive method for measuring protein concentration in subcellular membrane fractions prepared by sucrose density ultracentifugation. Anal Biochem 119:424-427.

Sockanathan S, Jessell TM (1998) Motor neuron-derived retinoid signalling specifies the subtype identity of spinal motor neurons. Cell 94:503-514.

Sperry RW (1963) Chemoaffinity in the orderly growth of nerve fiber patterns and connections. Proc Natl Acad Sci USA 50:703-710.

Stahl B, Muller B, von Boxberg Y, Cox EC, Bonhoeffer F (1990) Biochemical characterization of a putative axonal guidance molecule of the chick visual system. Neuron 5:735-743.

Swett JS, Eldred E, Buchwald JS (1970) Somatotopic cord-to-muscle relations in efferent innervation of cat gastrocnemius. Am J Physiol 219:762-766.

Tsuchida T, Ensini M, Morton SB, Baldassare M, Edlund T, Jessell TM, Pfaff SL (1994) Topographic organization of embryonic motor neurons defined by expression of LIM homeobox genes. Cell 79:957-970.

Walter J, Kern-Verts B, Huf J, Stolze B, Bonhoeffer F (1987a) Recognition of position-specific properties of tectal membranes by retinal axons in vitro. Development 101:685-696.

Walter J, Henke-Fahle S, Bonhoeffer F (1987b) Avoidance of posterior tectal membranes by temporal retinal axons in vitro. Development 101:909-913.

Wigston DJ, Sanes JR (1985) Selective reinnervation of intercostal muscles transplanted from different segmental levels to a common site. J Neurosci 5:1208-1221.

Xie HW, Ziskind-Conhaim L (1995) Blocking $\mathrm{Ca}^{2+}$-dependent synaptic release delays motoneuron differentiation in the rat spinal cord. J Neurosci 15:5900-5911. 